\title{
MECHANISMS OF STEROID ACTION AND RESISTANCE IN INFLAMMATION
}

\section{Corticosteroid-insensitive asthma: molecular mechanisms}

\author{
I M Adcock and S J Lane ${ }^{1}$ \\ Department of Thoracic Medicine, National Heart and Lung Institute, Imperial College London, Dovehouse Street, London SW3 6LY, UK \\ ${ }^{1}$ Department of Respiratory Medicine, Adelaide and Meath Hospital, Tallaght, Dublin 24, Eire \\ (Requests for offprints should be addressed to I M Adcock; Email: ian.adcock@imperial.ac.uk)
}

\begin{abstract}
Corticosteroids are the most potent anti-inflammatory agents used to treat chronic inflammatory diseases such as bronchial asthma. However, there are a small number $(<5 \%)$ of asthmatic patients who do not respond well, or at all, to corticosteroid therapy - the corticosteroidresistant and corticosteroid-dependent patients. Although this phenomenon is relatively uncommon, it poses a difficult therapeutic problem because few alternative therapies are available and these patients account for $>50 \%$ of the health care costs of asthma. If the mechanisms for corticosteroid insensitivity are understood they
\end{abstract}

may, in turn, provide insight into the key mechanism of corticosteroid action and allow a rational way to treat these individuals whose disease tends to be severe. Corticosteroid insensitivity is not limited to asthma and is a feature of other inflammatory diseases, such as rheumatoid arthritis and inflammatory bowel disease. Thus, elucidation of the cause for the relative lack of corticosteroid response in this subgroup of asthmatic individuals may have important implications for other diseases.

Journal of Endocrinology (2003) 178, 347-355

\section{The molecular basis of inflammation in bronchial asthma}

Inflammation is a central feature of many chronic lung diseases including bronchial asthma. The specific characteristics of the inflammatory response and the site of inflammation differ between these diseases, but all involve the recruitment and activation of inflammatory cells and changes in the structural cells of the lung. These diseases are characterised by an increased expression of many mediators involved in the inflammatory cascade, including cytokines, chemokines, growth factors, enzymes, receptors and adhesion molecules. Increased inflammatory gene transcription is regulated by pro-inflammatory transcription factors, such as nuclear factor- $\kappa \mathrm{B}(\mathrm{NF}-\kappa \mathrm{B})$ and activator protein-1 (AP-1). For example, NF- $\kappa B$ (Hart et al. 1998) and AP-1 (Demoly et al. 1992) are markedly activated in the epithelial cells of asthmatic patients and these transcription factors regulate many of the inflammatory genes that are abnormally expressed in asthma (Adcock \& Caramori 2001).

Alterations in the structure of chromatin are critical to the regulation of gene expression (Urnov \& Wolffe 2001). This chromatin structure is composed of nucleosomes which are particles consisting of $\sim 146$ bp DNA associated with an octomer of two molecules each of core histone proteins ( $\mathrm{H} 2 \mathrm{~A}, \mathrm{H} 2 \mathrm{~B}, \mathrm{H} 3$ and $\mathrm{H} 4)$. In the resting cell, DNA is tightly compacted around these basic core histones, excluding the binding of the enzyme RNA polymerase II, which activates the formation of mRNA. This conformation of the chromatin structure is described as closed and is associated with suppression of gene expression. Acetylation of lysine residues on histones induces a relaxed DNA structure allowing gene transcription to occur. Transcriptional co-activators such as cAMP response element binding protein (CREB)-binding protein (CBP) have intrinsic histone acetyltransferase (HAT) activity, which is further activated by the binding of transcription factors. Changes in the phosphorylation status of HATs also affect their activity. Increased gene transcription is therefore associated with an increase in histone acetylation, whereas hypo-acetylation is correlated with reduced transcription or gene silencing (Urnov \& Wolffe 2001).

\section{$N F-\kappa B$ and $A P-1$}

$\mathrm{NF}-\kappa \mathrm{B}$ is ubiquitously expressed and is able not only to control the induction of inflammatory genes in its own 
right but it can enhance the activity of other cell- and signal- specific transcription factors (Barnes \& Karin 1997). In addition, it is a major target for corticosteroids (Barnes \& Karin 1997). NF- $\kappa B$ is activated by all the stimuli thought to be important in asthma, including cytokines, such as tumour necrosis factor- $\alpha(\mathrm{TNF} \alpha)$ and interleukin (IL) $-1 \beta$, viruses and immune challenges (Barnes \& Karin 1997, Baldwin 2001). Activation of cell surface receptors leads to phosphorylation of receptor-associated kinases. These kinases, in turn, phosphorylate specific intracellular kinases (inhibitor of NF- $\mathrm{KB}$ kinase; IKK). Phosphorylation of IKKs results in phosphorylation of the NF- $\mathrm{KB}$ cytoplasmic inhibitor $(\mathrm{I}-\kappa \mathrm{B} \alpha)$, which targets $\mathrm{I}-\kappa \mathrm{B} \alpha$ for proteosomal degradation. This releases $\mathrm{NF}-\kappa \mathrm{B}$ from its inactive state, enabling nuclear translocation and binding to specific DNA response elements within the regulatory regions of responsive genes (Ghosh \& Karin 2002).

AP-1 is a transcription factor complex that is formed by dimerisation of members of the Fos (c-Fos, Fra1 and Fra2) and Jun (c-Jun, Jun B and Jun D) proto-oncogene families and is defined by binding to the phorbol ester 12-Otetradecanoylphorbol-13-acetate (TPA)-response element (TRE) (Chang \& Karin 2001, Shaulian \& Karin 2002). In the resting cell, AP-1 is composed of dimers of the Jun family and has weak DNA binding and gene transactivating activities. When the cell is activated, the components of AP-1 change rapidly to Fos:Jun heterodimers of which c-Fos:c-Jun is the most abundant and much more active than the resting homodimer. Inducible AP-1 is formed after activation of specific mitogen-activated protein kinases (MAPKs) of which Jun N-terminal kinase (JNK) is a central component (Chang \& Karin 2001). JNK increases AP-1's DNA binding and gene transactivating activity by increasing the production of c-Fos and by increasing the affinity of c-Jun for c-Fos. JNK also phosphorylates Ets-like kinase (Elk-1) which enhances c-Fos transcription (Chang \& Karin 2001) by binding to the serum response element in its promoter. c-Jun transcriptional activation is mediated by a TRE that is bound by the transcriptional activator activating transcription factor 2 (ATF-2), either as a homodimer or as a heterodimer with c-Jun. In this way, c-Jun may autoregulate expression of its own gene. In addition, ATF-2 is phosphorylated by JNK (Chang \& Karin 2001, Shaulian \& Karin 2002) leading to increased c-Jun expression.

\section{Glucocorticoid receptors}

Corticosteroids exert their effects by binding to a cytoplasmic receptor (glucocorticoid receptor; GR) (Adcock \& Caramori 2001). GRs are expressed in almost all cell types and are modular in structure. Thus, GR has several functional domains including a ligand-binding domain (LBD), a DNA-binding domain and two domains that are involved in transactivation of genes once binding to DNA has occurred via association with other proteins (activation function; AF-1 and AF-2). The second activation domain (AF-2) lies within the LBD. The inactive GR is bound to a protein complex that includes two subunits of the heat shock protein hsp90, which thus act as molecular chaperones preventing the nuclear localisation of unoccupied GR. Once the ligand binds to GR, hsp90 dissociates, allowing the nuclear localisation of the activated GRsteroid complex and its binding as a dimer to specific DNA sequences (glucocorticoid response elements (GREs); GGTACAnnnTGTTCT) and interaction with co-activator complexes (Adcock \& Caramori 2001).

\section{Glucocorticoid-induced gene transcription}

The number of genes per cell directly regulated by corticosteroids is estimated to be between 10 and 100, but many genes are indirectly regulated through an interaction with other transcription factors and co-activators. Corticosteroids may suppress inflammation by increasing the synthesis of anti-inflammatory proteins, such as annexin-1, IL-10, MAPK phosphatase-1 (MKP-1) and the inhibitor

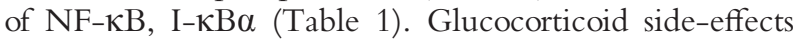
are manifold and their regulation, at the molecular level, involves both DNA-binding and non-DNA-binding events. It is likely that some side-effects, such as osteoporosis, glaucoma, growth retardation in children, wound healing and metabolic effects are mediated, at least in part, by DNA binding (Schacke et al. 2002a). GRs, as with other transcription factors, increase gene transcription through an action on chromatin remodelling and recruitment of RNA polymerase II to the site of local DNA unwinding (Karin 1998, Ito et al. 2000).

\section{Switching off inflammatory genes}

\section{Cross-talk between GR and other transcription factors}

The major anti-inflammatory effects of corticosteroids are thought to be due to repression of inflammatory and immune genes. The inhibitory effect of corticosteroids is due largely to protein-protein complex interactions between activated GR and transcription factors, such as $\mathrm{NF}-\kappa \mathrm{B}$ and AP-1, which mediate the expression of these inflammatory genes (Karin 1998) (Fig. 1). The interplay between pro-inflammatory transcription factors and GR may reflect differing effects on histone acetylation/ deacetylation (Ito et al. 2000).

The importance of cross-talk in GR actions is indicated by the construction of a GR dimerisation-deficient mutant mouse in which GR is unable to dimerise and therefore bind to DNA, thus separating the transactivation and transrepression activities of glucocorticoids (Reichardt et al. 1998). These animals, in contrast to GR knockout animals, survive to adulthood. In these animals, 
Table 1 Glucocorticoid-sensitive genes

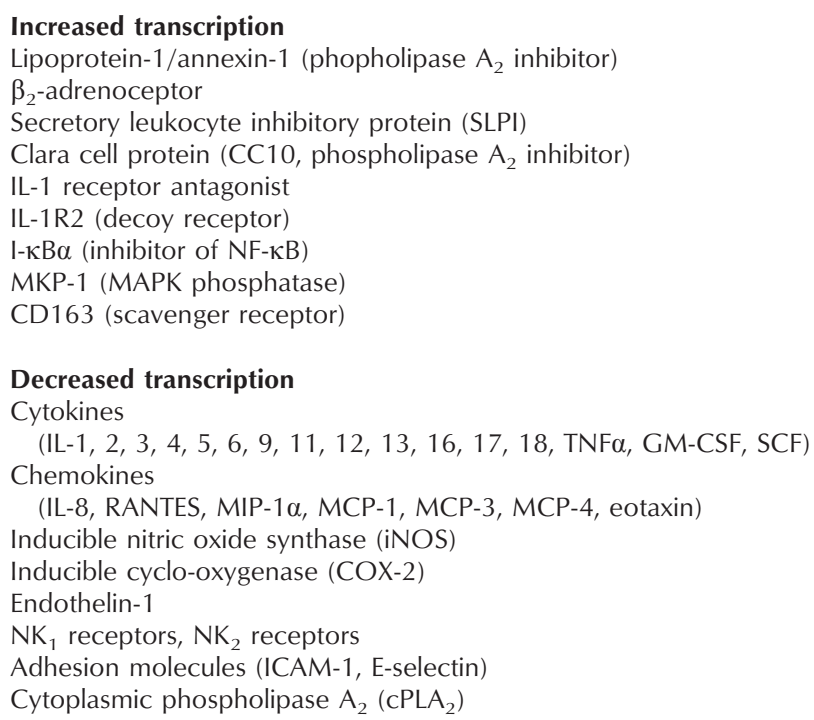

CD163, cluster differentiation 163; GM-CSF, granulocyte macrophage-cell stimulating factor; SCF, stem cell factor; RANTES, Regulated upon activation normal T-cell expressed and secreted; MIPI $\alpha$, macrophage inflammatory protein-1 $\alpha$; MCP, monocyte chemoattractant protein; NK, neurokinin; ICAM-I, intercellular adhesion molecule $\mathrm{I}$.

dexamethasone was able to inhibit AP-1- and NF-KBdriven gene transcription but the ability to facilitate GRE-mediated effects such as cortisol suppression and thymocyte apoptosis was markedly attenuated. This also suggests that the development of glucocorticoids with a greater therapeutic window is possible.

In addition, corticosteroids may also play a role in repressing the action of MAPKs such as the extracellular regulated kinase (ERK) and JNK (Rider et al. 1996, Caelles et al. 1997, Swantek et al. 1997, Hirasawa et al. 1998). Thus, Caelles and colleagues have demonstrated that corticosteroids inhibit the phosphorylation and activation of JNK, resulting in a failure to phosphorylate c-Jun and Elk-1, reduced c-fos transcription and a marked reduction in AP-1 activity. More recently it has been shown that dexamethasone can rapidly induce the dual specificity MAPK inhibitor MKP-1 and thereby attenuate p38 MAPK activation (Kassel et al. 2001, Lasa et al. 2001, 2002). Rogatsky et al. (1998) have, in turn, shown reciprocal inhibition of rat GR reporter gene activity by JNKs by a direct phosphorylation of serine 246 whereas ERK can inhibit GR action by an indirect effect, possibly through phosphorylation of a co-factor.

\section{Corticosteroid resistance}

Although corticosteroids are highly effective in the control of asthma and other chronic inflammatory or immune diseases, a small proportion of patients with asthma fail to respond even to high doses of oral corticosteroids (Leung \& Bloom 2003). Resistance to the therapeutic effects of corticosteroids is also recognised in other inflammatory and immune diseases (Lamberts et al. 1996), including rheumatoid arthritis (Lane \& Lee 1996) and inflammatory bowel disease (Hearing et al. 1999). Corticosteroidresistant (CR) patients, although uncommon, present considerable management problems. It is likely that there is a spectrum of steroid responsiveness, with the rare resistance at one end, but a relative resistance is seen in patients who require high doses of inhaled and oral corticosteroids (corticosteroid-dependent asthma; CD) (Leung \& Bloom 2003).

CR asthma has been defined as a failure of the forced expired volume in $1 \mathrm{~s}$ to improve from a baseline value of $\leq 75 \%$ predicted by $\geq 15 \%$ after 14 days of treatment with $40 \mathrm{mg}$ prednisolone orally, despite demonstrating $>15 \%$ reversibility to an inhaled $\beta_{2}$ agonist (Barnes et al. 1995). In addition to reduced changes in clinical symptoms following corticosteroid therapy, studies have shown that there is reduced suppression of IL- 4 and IL- 5 mRNA in bronchoalveolar lavage cells obtained from CR patients after 1 week of treatment with prednisolone, when compared with those of corticosteroid-sensitive (CS) asthmatic subjects (Leung et al. 1995). Bronchoalveolar lavage of a group of $\mathrm{CR}$ subjects revealed an increased number of cells expressing IL-2, IL-4 and IL-13 mRNA compared with CS asthmatics (Leung et al. 1995). This suggested 


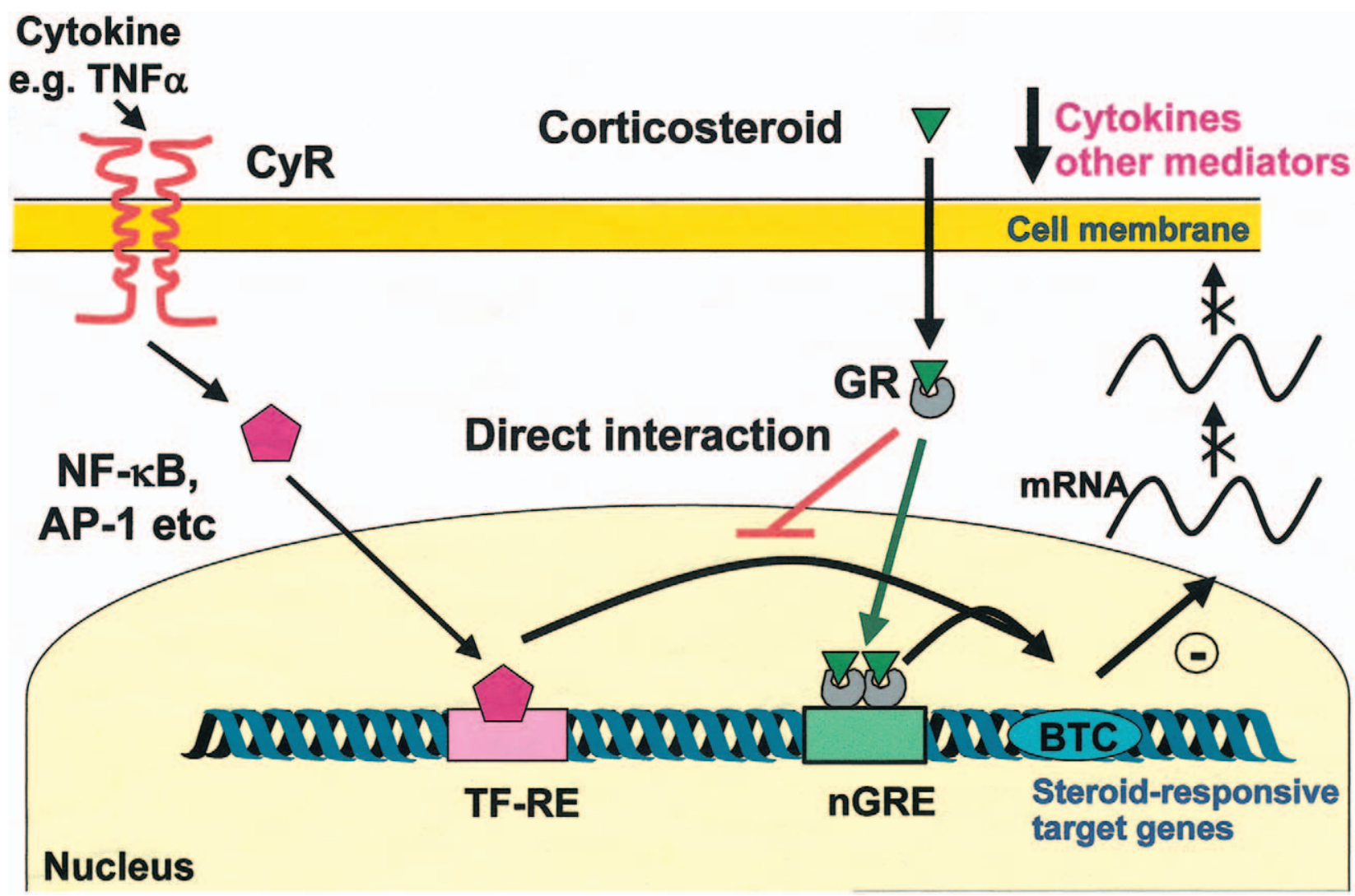

Figure 1 How glucocorticoids switch off inflammatory genes. Inflammatory genes are activated by inflammatory stimuli, such as IL-1 $\beta$ or $\mathrm{TNF} \alpha$, acting through their receptors (CyR), resulting in activation of the transcription factors NF- $\mathrm{B}$ and AP-1. Upon activation, these are able to bind to specific recognition sites within the promoter regions of responsive genes (TF-RE) and stimulate transcription of inflammatory genes such as cytokines and other mediators following recruitment of the basal transcription complex (BTC). GRs, after activation by corticosteroids, translocate to the nucleus and bind to either a negative GR response element (nGRE) in the promoter of inflammatory genes inhibiting gene transcription or, more commonly, interact with and block the ability of AP-1 and NF-kB from enhancing gene expression.

that the profile of cytokine expression may underlie the poor responsiveness to glucocorticoids in these patients.

Importantly for examining the molecular basis of corticosteroid insensitivity, CR asthma is also associated with impaired in vitro and in vivo responsiveness of peripheral blood mononuclear cells (PBMCs) to the suppressive effects of corticosteroids. Thus, in patients with CR and $\mathrm{CD}$ asthma there is a reduction in the inhibitory effect of corticosteroids on cytokine release in PBMCs (Dong et al. 1998, Kam et al. 1993, Leung \& Bloom 2003 and references therein).

\section{Molecular mechanisms of corticosteroid resistance}

At a molecular level, resistance to the anti-inflammatory effects of glucocorticoids can be induced by several mechanisms. The reduction in corticosteroid responsiveness observed in cells from these subjects has been ascribed to a reduced number of GR, altered affinity of the ligand for GR, reduced ability of the GR to bind to DNA or increased expression of inflammatory transcription factors, such as AP-1, that compete for DNA binding (Dong et al. 1998, Kam et al. 1993, Leung \& Bloom 2003).

\section{Defects in GR sequence and pharmacokinetics}

Unlike familial corticosteroid resistance where there is a mutation in the LBD of GR and a subsequent resetting of the basal cortisol level, CR patients have normal cortisol levels and are not Addisonian (Malchoff et al. 1993). Using standard dexamethasone suppression tests, it has been shown that $\mathrm{CR}$ asthmatics do not have an altered secretory rate of endogenous cortisol or an altered sensitivity of the hypothalamic-pituitary-adrenal axis (Lane et al. 1996). Using chemical mutational analysis, no mutations in the GR of CR patients were observed (Lane et al. 1994). This was confirmed in a later study which used RT-PCR 
(Adcock et al. 1995b). It is unlikely, therefore, that the defect in CR asthma lies in the structure of the GR.

\section{Defects in ligand binding}

We, and others, have previously demonstrated using whole cell binding assays no significant changes in monocyte and T-cell binding affinity $\left(K_{\mathrm{d}}\right)$ and receptor density of the GR in patients with CR asthma (Corrigan et al. 1991, Lane \& Lee 1991). More recently, Sher et al. (1994) have described two patterns of ligand-binding abnormalities in CR asthmatics termed type 1 and 2 . The more common type 1 defect was associated with reduced $K_{\mathrm{d}}$ of GR, normal receptor numbers and was specific to $\mathrm{T}$ cells. The less common type 2 defect was associated with reduced GR receptor density with a normal $K_{\mathrm{d}}$ and was seen in the total mononuclear cell population. These differences were detected only in the nucleus and not the cytoplasm, possibly reflecting an effect of a nuclear protein masking the GR ligand-binding site or in an altered conformation of the activated GR. This altered affinity of dexamethasone for GR may reflect either an intrinsic defect in the GR within these patients or may relate to changes in the receptor induced by the increased level of inflammation in more severe asthmatics. The reversal of the reduced binding affinity by incubation with normal media suggests that the latter is a more likely possibility (Irusen et al. 2002, Leung \& Bloom 2003). The type 1 defect was reversible with serum deprivation and was mimicked by incubation of cells with high concentrations of IL-2 and IL-4 or by IL-13 alone (Irusen et al. 2002, Leung \& Bloom 2003). In contrast, the type 2 defect was irreversible and was not IL-2 and IL-4 dependent (Sher et al. 1994).

Two explanations for the effect of IL-2/IL-4 or IL-13 alone on ligand-binding characteristics have been proposed. Leung \& Bloom (2003) have associated these changes with an increased expression of the dominant negative isoform of GR, GR $\beta$, although others have been unable to detect enhanced GR $\beta$ expression in PBMCs from these CR patients (Gagliardo et al. 2000, Irusen et al. 2002). In contrast, increased numbers of cells expressing GR $\beta$ have been reported in skin biopsies from CR patients (Sousa et al. 2000). We have recently demonstrated that the effects of IL-2/IL-4 and IL-13 on GRligand binding and dexamethasone regulation of IL-10 release were blocked by the p38 MAPK inhibitor SB203580. Activation of p38 MAPK by IL-2/IL-4 resulted in serine phosphorylation of GR and reduced dexamethasone repression of lipopolysaccharide (LPS)stimulated GM-CSF release. The ability of dexamethasone to modulate IL-10 release was also inhibited by IL-2/IL-4 co-treatment and restored by SB203580 (Irusen et al. 2002). These data showed that $\mathrm{p} 38$ MAPK inhibitors may have potential in reversing glucocorticoid insensitivity and re-establishing the beneficial effects of glucocorticoids in patients with severe asthma.

It is unclear whether this is a direct or indirect effect of p38 MAPK or whether GR phosphorylation alters ligand binding affinity directly. This may result from either a change in GR conformation due to association of distinct co-factors, or partial blocking of the ligand-binding domain due to association of GR with nuclear transcriptional modulating proteins. Similar results have been seen following $\mathrm{NO}$ treatment of GR, whereby nitrosylation of GR at an hsp90 interaction site modified ligand binding (Galigniana et al. 1999). Serine 226 and the sequences immediately surrounding it are highly conserved, suggesting that its phosphorylation may alter or disrupt the protein-protein interactions regulating GR action.

\section{GR nuclear translocation and GR/GRE binding}

In one subgroup of CR and CD patients, nuclear localisation of GR in response to a high concentration $\left(10^{-6} \mathrm{M}\right)$ of dexamethasone was impaired (Matthews et al. 2000). The mechanism for this effect is unclear but may reflect changes in GR phosphorylation by MAPK and subsequent interaction with importin- $\alpha$ (Rogatsky et al. 1998, Savory et al. 1999, Irusen et al. 2002). This may also explain the earlier results we obtained using (Electrophoretic mobility shift assays (EMSAs) which showed that CR patients had a reduced level of GR:GRE binding compared with CS and non-asthmatic individuals following stimulation of PBMCs with dexamethasone (Adcock et al. 1995b). Scatchard analysis of GR:GRE binding showed no change in binding affinity but did show a reduced number of GR available for DNA binding in the CR patients. These results suggest that the ability of GR to bind to GRE is impaired in CR patients because of a reduced number of GR (Adcock et al. 1995b).

In a separate subgroup of CR patients, GR nuclear translocation was normal but dexamethasone could not correctly stimulate histone $\mathrm{H} 4$ acetylation (Matthews et al. 2000). This suggests that corticosteroids are not able to activate certain genes that are critical to the antiinflammatory action of high doses of corticosteroids. The mechanism for this effect is unknown but may reflect the mutual inhibitory effects of excess JNK activation (Rogatsky et al. 1998) in these cells or a failure of GR to recruit specific co-activators.

\section{Cross-talk with other transcription factors}

We originally reported an increase in the basal levels of AP-1 DNA binding in the nuclei from CR patients although no differences in the sequences of $c-f o s$ and $c-j u n$ mRNA were detected. In addition, there was a reduced ability of GR to interact and repress AP-1 activity (Adcock et al. 1995a). It is also possible to see enhanced c-Fos expression in bronchial biopsies of CR patients (Fig. 2). 


\section{C-Fos}
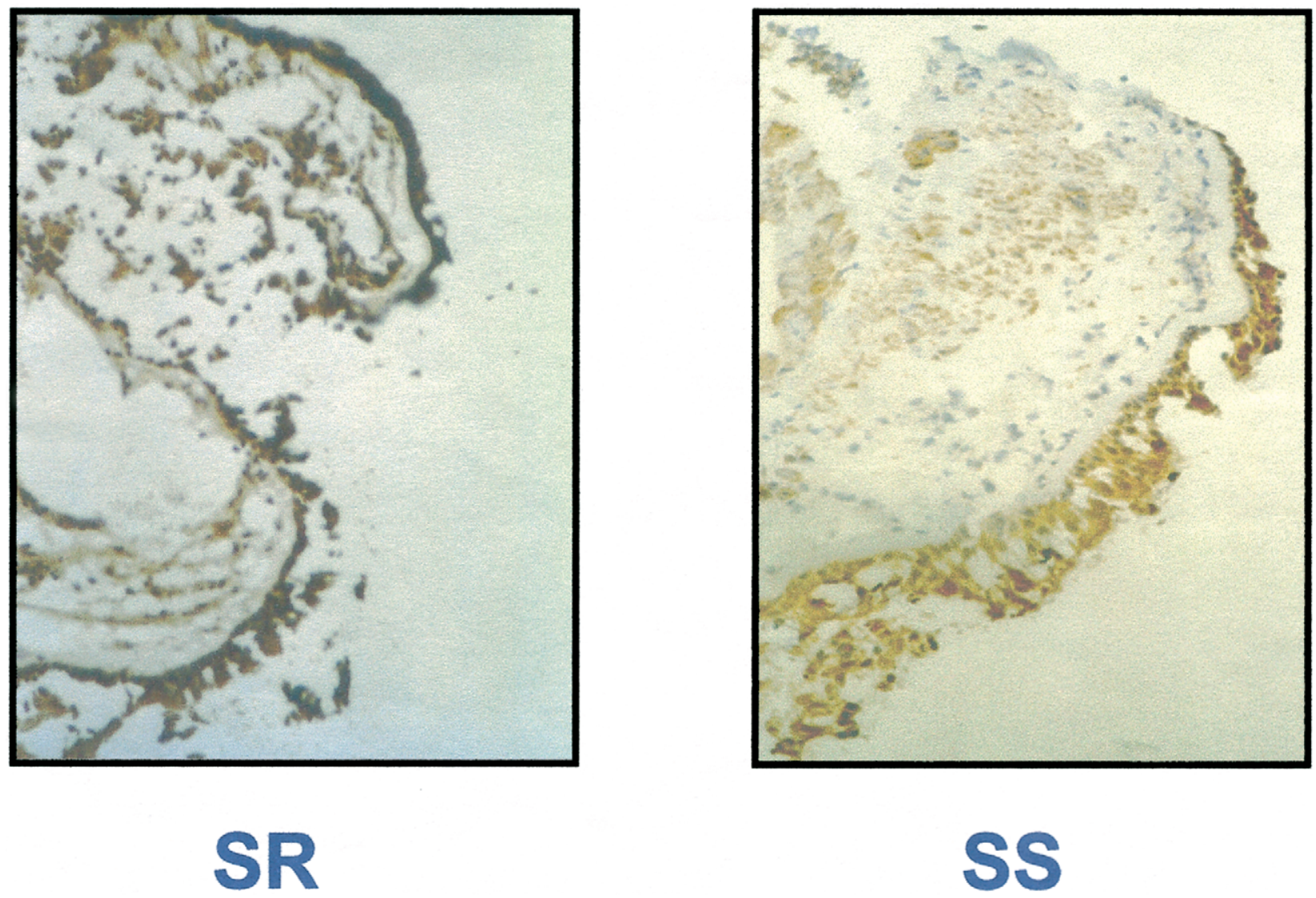

Figure 2 Enhanced expression of c-Fos in the bronchial airways of steroid (corticosteroid)-resistant (SR) asthmatics compared with steroid-sensitive subjects (SS). Increased intensity of dark brown immunohistological staining of c-Fos within the airway epithelium and infiltrating mononuclear cells in SR compared with SS subjects.

These results suggested that AP-1 is altered in CR patients and that increased levels of AP-1 may prevent GR function.

In a subsequent study using nuclear run-on, RT-PCR and Western blotting, we demonstrated a two- to fourfold greater increase in the c-fos transcription rate and mRNA and protein expression in PBMCs isolated from CR compared with CS asthmatics and normal subjects (Lane et al. 1998). When cells were stimulated with phorbol 12-myristate 13-acetate (PMA), the time- and concentration-dependent induction of c-Fos was greater in the $\mathrm{CR}$ group. Overexpression of c-Fos induced by stimulation of PBMCs derived from CS subjects with PMA for $6 \mathrm{~h}$ attenuated the ability of these cells to induce GR-GRE binding after $1 \mathrm{~h}$ of dexamethasone treatment. In these experiments, GR-GRE binding was reduced to levels similar to those seen in CR subjects. Incubation of PBMCs derived from CR subjects with dexamethasone and with antisense oligonucleotides directed against c-fos increased GR-GRE binding to levels similar to those seen in CS individuals. These findings suggested that increased c-Fos under basal conditions is the predominant inhibitory activity on GR-DNA binding in CR asthma.

The results of these studies did not determine whether there is a specific abnormality in the activation of $\mathrm{c}-\mathrm{fos}_{\mathrm{s}}$ transcription in PBMCs derived from $\mathrm{CR}$ subjects or a more generalised activation of the components of AP-1 or their regulatory pathways which activate components of AP-1 through the serum response element (Shaulian \& Karin 2002). Using the tuberculin response as a model of mononuclear cell inflammation, Sousa et al. (1999) subsequently showed a marked increase in the expression of activated phosphorylated c-Jun, enhanced expression of JNK, and greater up-regulation of c-Fos expression in the CR compared with the CS group. In this model, prednisolone suppressed memory T-cell, macrophage and 


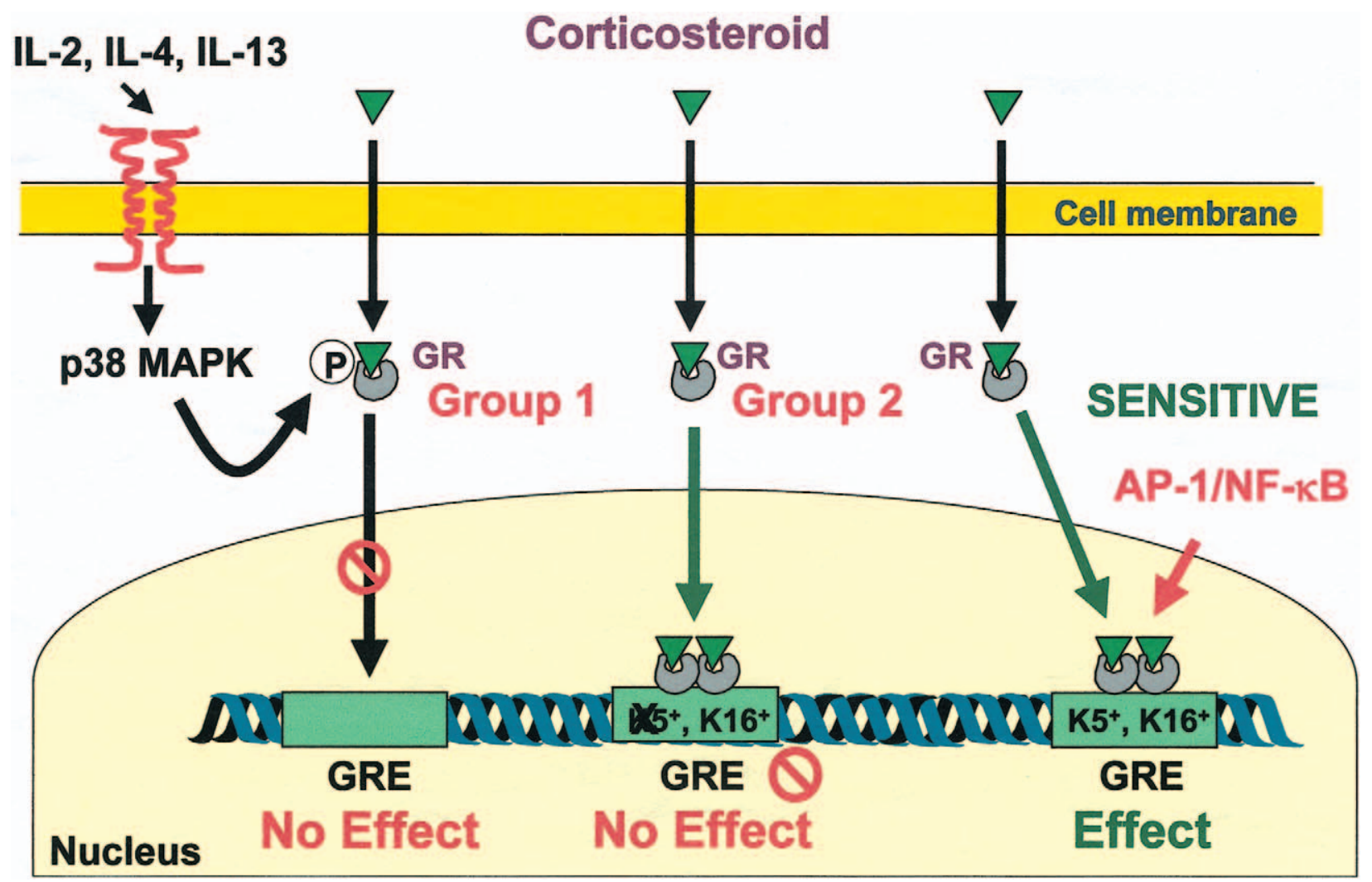

Figure 3 Potential mechanisms for corticosteroid insensitivity. Corticosteroids are lipophilic molecules that diffuse readily through cell membranes to interact with cytoplasmic receptors. Upon ligand binding, receptors (GR) are activated and translocate into the nucleus where they bind to specific DNA elements. Upon DNA binding, they induce acetylation of lysine residues $5\left(\mathrm{~K}^{+}\right)$and $16\left(\mathrm{~K} 16^{+}\right)$on histone $\mathrm{H} 4$ leading to modulation of gene transcription. Alternatively, GR can repress NF-KB- or AP-1-induced gene transcription. In some steroid-insensitive subjects (Group 1) GR is unable to translocate to the nucleus, possibly as a result of p38 MAPK-induced phosphorylation, and is thereby ineffective. In other steroid-insensitive subjects (Group 2), although GR can successfully translocate to the nucleus, it is unable to inhibit NF-KB and AP-1 and, in addition, has a reduced ability to induce histone acetylation on K5.

activated eosinophil infiltration into tuberculin-induced skin lesions of CS but not CR individuals. Prednisolone reduced the levels of both phosphorylated c-Jun and phosphorylated JNK in the CS but not the CR group without affecting total c-Jun and JNK expression.

The data to date suggest that increased levels of c-Fos and increased activation of c-Jun in patients with $\mathrm{CR}$ asthma account for the increased AP-1 activity seen in vitro and probably relates to increased activation of JNK in these subjects. JNK regulates the expression and activation of both major components of AP-1. Elevated JNK activity could be critical to the mechanisms of CR asthma and failure to inhibit JNK phosphorylation by glucocorticoids may be a major cause for the lack of response to glucocorticoids in CR asthma. In addition, JNK may, in turn, suppress GR function, resulting in a feed-forward loop of increasing inflammation and reduced corticosteroid responsiveness in these patients.

It is unclear whether increased c-fos transcription and JNK activation is a primary or secondary defect caused by excessive production of a unique pattern of cytokines in asthmatic airways. At present there is no evidence for a genetic component leading to enhanced AP-1 activation in $\mathrm{CR}$ asthma. The increased numbers of bronchoalveolar lavage cells expressing IL-2 and IL-4 in the CR group may suggest a primary defect of cytokine regulation in these patients. T-helper $2\left(\mathrm{TH}_{2}\right)$ cytokines can enhance AP-1 expression (Wang et al. 1994) which, in turn, can switch on more $\mathrm{TH}_{2}$ cytokines (de Groot et al. 1997), leading to a pro-inflammatory amplification loop. Irrespective of whether enhanced expression of AP-1 is primary or secondary, the net result is an excessive accumulation of this critical transcription factor.

\section{Therapeutic implications}

Inhaled glucocorticoids are now used as first-line therapy for the treatment of persistent asthma in adults and children in many countries, as they are the most effective 
treatments for asthma currently available (Barnes 1995). However, at high doses systemic absorption of inhaled glucocorticoids may have deleterious effects, so there has been a search for safer glucocorticoids for inhalation and oral administration. This has led to a search for novel glucocorticoids that selectively transrepress without significant transactivation, thus reducing the potential risk of systemic side-effects.

Recently, a novel class of glucocorticoids has been described in which there is potent transrepression with relatively little transactivation. These 'dissociated' glucocorticoids, including RU24858 and RU40066 have anti-inflammatory effects in vitro (Vayssiere et al. 1997), although there is little separation of anti-inflammatory effects and systemic side-effects in vivo (Belvisi et al. 2001). This may reflect in vivo metabolism of the glucocorticoids. Several non-steroidal selective glucocorticoid receptor agonists (SEGRA) have recently been reported that show dissociated properties in human cells (Schacke et al. 2002b). Several of these dissociated glucocorticoids and SEGRA are now in clinical development and show good separation between transrepression and transactivation actions. This suggests that the development of glucocorticoids and SEGRA with a greater margin of safety is possible and may even lead to the development of oral compounds that do not have significant adverse effects. Alternatively, it may be possible to use MAPK inhibitors as steroid-sparing agents reducing the dose of corticosteroid needed to obtain effective therapy.

\section{Conclusions}

CR asthma is a syndrome of relative corticosteroid insensitivity, without a clear single pathophysiological cause, rather than a distinct disease with complete resistance to corticosteroids. Several mechanisms have been proposed to account for a failure to respond to corticosteroids (Fig. 3), including a reduced number of GR, altered affinity of the ligand for GR, reduced ability of the GR to bind to DNA or increased activation of transcription factors, such as AP-1, that compete for DNA binding. These events may not be completely exclusive, in that MAPK activation may lead to enhanced inflammation, reduced GR ligand and DNA binding and possibly enhanced GR $\beta$ expression. The development of new dissociated corticosteroids may allow high enough doses of corticosteroids to be given to these patients to elicit therapeutic responses bypassing the problem of the deleterious side-effects normally seen in these patients. In addition, MAPK inhibitors may prove to be beneficial as corticosteroid-sparing agents.

\section{References}

Adcock IM \& Caramori G 2001 Cross-talk between pro-inflammatory transcription factors and glucocorticoids. Immunology and Cell Biology 79 376-384.
Adcock IM, Lane SJ, Brown CR, Lee TH \& Barnes PJ 1995a Abnormal glucocorticoid receptor-activator protein 1 interaction in steroid-resistant asthma. Journal of Experimental Medicine $\mathbf{1 8 2}$ 1951-1958.

Adcock IM, Lane SJ, Brown CR, Peters MJ, Lee TH \& Barnes PJ $1995 b$ Differences in binding of glucocorticoid receptor to DNA in steroid-resistant asthma. Journal of Immunology 154 3500-3505.

Baldwin AS Jr 2001 Series introduction: the transcription factor NF-kappaB and human disease. Journal of Clinical Investigation 107 3-6.

Barnes PJ 1995 Inhaled glucocorticoids for asthma. New England Journal of Medicine 332 868-875.

Barnes PJ \& Karin M 1997 Nuclear factor-kappaB: a pivotal transcription factor in chronic inflammatory diseases. New England Journal of Medicine 336 1066-1071.

Barnes PJ, Greening AP \& Crompton GK 1995 Glucocorticoid resistance in asthma. American Journal of Respiratory and Critical Care Medicine 152 S125-S140.

Belvisi MG, Wicks SL, Battram CH, Bottoms SE, Redford JE, Woodman P, Brown TJ, Webber SE \& Foster ML 2001 Therapeutic benefit of a dissociated glucocorticoid and the relevance of in vitro separation of transrepression from transactivation activity. Journal of Immunology 166 1975-1982.

Caelles C, Gonzalez-Sancho JM \& Munoz A 1997 Nuclear hormone receptor antagonism with AP-1 by inhibition of the JNK pathway. Genes and Development 11 3351-3364.

Chang L \& Karin M 2001 Mammalian MAP kinase signalling cascades. Nature 410 37-40.

Corrigan CJ, Brown PH, Barnes NC, Szefler SJ, Tsai JJ, Frew AJ \& Kay AB 1991 Glucocorticoid resistance in chronic asthma. Glucocorticoid pharmacokinetics, glucocorticoid receptor characteristics, and inhibition of peripheral blood $\mathrm{T}$ cell proliferation by glucocorticoids in vitro. American Reviews in Respiratory Disease 144 1016-1025.

Demoly P, Basset SN, Chanez P, Campbell AM, Gauthier RC, Godard P, Michel FB \& Bousquet J 1992 c-fos proto-oncogene expression in bronchial biopsies of asthmatics. American Journal of Respiratory and Cell Molecular Biology 7 128-133.

Dong Y, Poellinger L, Gustafsson JA \& Okret S 1988 Regulation of glucocorticoid receptor expression: evidence for transcriptional and posttranslational mechanisms. Molecular Endocrinology 2 1256-1264.

Gagliardo R, Chanez P, Vignola AM, Bousquet J, Vachier I, Godard P, Bonsignore G, Demoly P \& Mathieu M 2000 Glucocorticoid receptor alpha and beta in glucocorticoid dependent asthma. American Journal of Respiratory and Critical Care Medicine 162 7-13.

Galigniana MD, Piwien-Pilipuk G \& Assreuy J 1999 Inhibition of glucocorticoid receptor binding by nitric oxide. Molecular Pharmacology 55 317-323.

Ghosh S \& Karin M 2002 Missing pieces in the NF-kappaB puzzle. Cell 109 (Suppl) S81-S96.

De Groot RP, van Dijk TB, Caldenhoven E, Coffer PJ, Raaijmakers JA, Lammers JW \& Koenderman L 1997 Activation of 12-Otetradecanoylphorbol-13-acetate response element-and dyad symmetry element-dependent transcription by interleukin-5 is mediated by Jun $\mathrm{N}$-terminal kinase/stress-activated protein kinase kinases. Journal of Biological Chemistry 272 2319-2325.

Hart LA, Krishnan VL, Adcock IM, Barnes PJ \& Chung KF 1998 Activation and localization of transcription factor, nuclear factorkappaB, in asthma. American Journal of Respiratory and Critical Care Medicine 158 1585-1592.

Hearing SD, Norman M, Probert CS, Haslam N \& Dayan CM 1999 Predicting therapeutic outcome in severe ulcerative colitis by measuring in vitro steroid sensitivity of proliferating peripheral blood lymphocytes. Gut 45 382-388.

Hirasawa N, Sato Y, Fujita Y, Mue S \& Ohuchi K 1998 Inhibition by dexamethasone of antigen-induced c-Jun $\mathrm{N}$-terminal kinase activation in rat basophilic leukemia cells. Journal of Immunology 161 4939-4943. 
Irusen E, Matthews JG, Takahashi A, Barnes PJ, Chung KF \& Adcock IM 2002 p38 mitogen-activated protein kinase-induced glucocorticoid receptor phosphorylation reduces its activity: role in steroid-insensitive asthma. Journal of Allergy and Clinical Immunology 109 649-657.

Ito K, Barnes PJ \& Adcock IM 2000 Glucocorticoid receptor recruitment of histone deacetylase 2 inhibits interleukin-1 betainduced histone $\mathrm{H} 4$ acetylation on lysines 8 and 12. Molecular Cell Biology 20 6891-6903.

Kam JC, Szefler SJ, Surs W, Sher ER \& Leung DY 1993 Combination IL-2 and IL-4 reduces glucocorticoid receptor-binding affinity and $\mathrm{T}$ cell response to glucocorticoids. Journal of Immunology $1513460-3466$.

Karin M 1998 New twists in gene regulation by glucocorticoid receptor: is DNA binding dispensable? Cell $\mathbf{9 3}$ 487-490.

Kassel O, Sancono A, Kratzschmar J, Kreft B, Stassen M \& Cato AC 2001 Glucocorticoids inhibit MAP kinase via increased expression and decreased degradation of MKP-1. EMBO Journal 20 7108-7116.

Lamberts SW, Huizenga AT, de LP, de JF \& Koper JW 1996 Clinical aspects of glucocorticoid sensitivity. Steroids 61 157-160.

Lane SJ \& Lee TH 1991 Glucocorticoid receptor characteristics in monocytes of patients with corticosteroid-resistant bronchial asthma. American Review of Respiratory Disease 143 1020-1024.

Lane SJ \& Lee TH 1996 Corticosteroid resistance in other disease states and tissues. American Journal of Respiratory and Critical Care Medicine 154 S62-S65.

Lane SJ, Arm JP, Staynov DZ \& Lee TH 1994 Chemical mutational analysis of the human glucocorticoid receptor cDNA in glucocorticoid-resistant bronchial asthma. American Journal of Respiratory Cell and Molecular Biology 11 42-48.

Lane SJ, Atkinson BA, Swaminathan R \& Lee TH 1996 Hypothalamic-pituitary-adrenal axis in corticosteroid-resistant bronchial asthma. American Journal of Respiratory and Critical Care Medicine 153 557-560.

Lane SJ, Adcock IM, Richards D, Hawrylowicz C, Barnes PJ \& Lee TH 1998 Corticosteroid-resistant bronchial asthma is associated with increased $\mathrm{c}$-fos expression in monocytes and $\mathrm{T}$ lymphocytes. Journal of Clinical Investigation 102 2156-2164.

Lasa M, Brook M, Saklatvala J \& Clark AR 2001 Dexamethasone destabilizes cyclooxygenase 2 mRNA by inhibiting mitogenactivated protein kinase p38. Molecular and Cellular Biology 21 771-780.

Lasa M, Abraham SM, Boucheron C, Saklatvala J \& Clark AR 2002 Dexamethasone causes sustained expression of mitogen-activated protein kinase (MAPK) phosphatase 1 and phosphatase-mediated inhibition of MAPK p38. Molecular and Cellular Biology 22 7802-7811.

Leung DY \& Bloom JW 2003 Update on glucocorticoid action and resistance. Journal of Allergy and Clinical Immunology 111 3-22.

Leung DY, Martin RJ, Szefler SJ, Sher ER, Ying S, Kay AB \& Hamid Q 1995 Dysregulation of interleukin 4, interleukin 5, and interferon gamma gene expression in steroid-resistant asthma. Journal of Experimental Medicine 181 33-40.

Malchoff DM, Brufsky A, Reardon G, McDermott P, Javier EC, Bergh CH, Rowe D \& Malchoff CD 1993 A mutation of the glucocorticoid receptor in primary cortisol resistance. Journal of Clinical Investigation 91 1918-1925.

Matthews JG, Ito K, Barnes PJ \& Adcock IM 2000 Corticosteroidresistant and corticosteroid-dependent asthma: two clinical phenotypes can be associated with the same in vitro defects in GR nuclear translocation and acetylation of histone H4. American Journal of Respiratory and Critical Care Medicine 161 A189.

Reichardt HM, Kaestner KH, Tuckermann J, Kretz O, Wessely O, Bock R, Gass P, Schmid W, Herrlich P, Angel P \& Schutz G 1998 DNA binding of the glucocorticoid receptor is not essential for survival. Cell 93 531-541.

Rider LG, Hirasawa N, Santini F \& Beaven MA 1996 Activation of the mitogen-activated protein kinase cascade is suppressed by low concentrations of dexamethasone in mast cells. Journal of Immunology 157 2374-2380.

Rogatsky I, Logan SK \& Garabedian MJ 1998 Antagonism of glucocorticoid receptor transcriptional activation by the c-Jun N-terminal kinase. PNAS 95 2050-2055.

Savory JA, Hsu B, Laquian IR, Giffin W, Reich T, Hache RG \& Lefebvre YA 1999 Discrimination between NL1- and NL2mediated nuclear localization of the glucocorticoid receptor. Molecular and Cellular Biology 19 1025-1037.

Schacke H, Docke WD \& Asadullah K 2002a Mechanisms involved in the side effects of glucocorticoids. Pharmacology and Therapeutics $9623-43$.

Schacke H, Hennekes H, Schottelius A, Jaroch S, Lehmann M, Schmees N, Rehwinkel H \& Asadullah K 2002b SEGRAs: a novel class of anti-inflammatory compounds. Ernst Schering Research Foundation Workshop 40 357-371.

Shaulian E \& Karini M 2002 AP-1 as a regulator of cell life and death. Nature Cell Biology 4 E131-E136.

Sher ER, Leung DY, Surs W, Kam JC, Zieg G, Kamada AK \& Szefler SJ 1994 Steroid-resistant asthma. Cellular mechanisms contributing to inadequate response to glucocorticoid therapy. Journal of Clinical Investigation 93 33-39.

Sousa AR, Lane SJ, Soh C \& Lee TH 1999 In vivo resistance to corticosteroids in bronchial asthma is associated with enhanced phosyphorylation of JUN N-terminal kinase and failure of prednisolone to inhibit JUN N-terminal kinase phosphorylation. Journal of Allergy and Clinical Immunology 104 565-574.

Sousa AR, Lane SJ, Cidlowski JA, Staynov DZ \& Lee TH 2000 Glucocorticoid resistance in asthma is associated with elevated in vivo expression of the glucocorticoid receptor beta-isoform. Journal of Allergy and Clinical Immunology 105 943-950.

Swantek JL, Cobb MH \& Geppert TD 1997 Jun N-terminal kinase/stress-activated protein kinase (JNK/SAPK) is required for lipopolysaccharide stimulation of tumor necrosis factor alpha (TNF-alpha) translation: glucocorticoids inhibit TNF-alpha translation by blocking JNK/SAPK. Molecular and Cellular Biology 17 6274-6282.

Urnov FD \& Wolffe AP 2001 Chromatin remodeling and transcriptional activation: the cast (in order of appearance). Oncogene 20 2991-3006

Vayssiere BM, Dupont S, Choquart A, Petit F, Garcia T, Marchandeau C, Gronemeyer H \& Resche RM 1997 Synthetic glucocorticoids that dissociate transactivation and AP-1 transrepression exhibit antiinflammatory activity in vivo. Molecular Endocrinology 11 1245-1255.

Wang CY, Bassuk AG, Boise LH, Thompson CB, Bravo R \& Leiden JM 1994 Activation of the granulocyte-macrophage colonystimulating factor promoter in $\mathrm{T}$ cells requires cooperative binding of Elf-1 and AP-1 transcription factors. Molecular and Cellular Biology 14 1153-1159.

Received 6 March 2003

Accepted 23 June 2003 